\title{
Electrostatic Equilibrium of Two Spherical Charged Masses in General Relativity
}

\author{
G. P. Perry and F. I. Cooperstock \\ Department of Physics and Astronomy, University of Victoria \\ P.O. Box 3055, Victoria, B.C. V8W 3P6 (Canada)
}

(October 30, 2018)

\begin{abstract}
Approximate solutions representing the gravitational-electrostatic balance of two arbitrary point sources in general relativity have led to contradictory arguments in the literature with respect to the condition of balance. Up to the present time, the only known exact solutions which can be interpreted as the non-linear superposition of two spherically symmetric (Reissner-Nordström) bodies without an intervening strut has been for critically charged masses, $M_{i}^{2}=Q_{i}^{2}$. In the present paper, an exact electrostatic solution of the EinsteinMaxwell equations representing the exterior field of two arbitrary charged Reissner-Nordström bodies in equilibrium is studied. The invariant physical charge for each source is found by direct integration of Maxwell's equations. The physical mass for each source is invariantly defined in a manner similar to which the charge was found. It is shown through numerical methods that balance without tension or strut can occur for non-critically charged bodies. It is demonstrated that other authors have not identified the correct physical parameters for the mass and charge of the sources. Further properties of the solution, including the multipole structure and comparison with other parameterizations, are examined.
\end{abstract}

04.20.Jb, 04.40.Nr 


\section{INTRODUCTION}

In a recent paper by Bonnor [1], the equilibrium conditions for a charged test particle in the field of a spherically symmetric charged mass (Reissner-Nordström solution) were investigated. He found that the classical condition for equilibrium

$$
M_{1} M_{2}=Q_{1} Q_{2}
$$

for which the separation between the particles is arbitrary, was neither necessary nor sufficient for electrostatic balance of two spherical masses. This is in conflict with the earlier results of Barker and O'Connell [2] and Ohta and Kimura [3] who used different approximation methods. Barker and O'Connell claimed that in the post-Newtonian approximation, the equation

$$
\left(M_{1} Q_{2}-M_{2} Q_{1}\right)\left(Q_{1}-Q_{2}\right)=0
$$

had to be satisfied in addition to (1.1). Ohta and Kimura claimed that in the post-postNewtonian approximation, the necessary and sufficient condition for balance is that each mass be "critically" charged,

$$
M_{i}=\left|Q_{i}\right| \quad i=1,2
$$

and balance can occur for arbitrary separation of the sources. Up to the present time, the problem of gravitational-electrostatic balance of two spherical bodies in general relativity without an intervening Weyl line singularity (strut or tension) has been solved exactly only for critically charged masses $[4$ [ 6]. A balance solution was originally thought to have been found [7] within the Herlt class for both sources having $M_{i}>\left|Q_{i}\right|$, but it was subsequently shown that the intervening line singularity could not be removed [8]. Kramer [9] presented an exact solution for the electrostatic counterpart of the double Kerr-NUT solution with zero spin parameter. He found that condition (1.1) holds for electrostatic balance. However he stated that his solution cannot be interpreted as the non-linear superposition of two Reissner-Nordström solutions and thus the masses are not spherically symmetric.

In the present paper, an exact electrostatic solution of the Einstein-Maxwell equations representing the exterior field of two arbitrary charged non-linearly superposed ReissnerNordström sources in equilibrium is given. It is obtained with the aid of Sibgatullin's [10] method for constructing the complex Ernst potentials [11]. It is mathematically equivalent to the solutions of Manko et al [12] and Chamorro et al [13], henceforth referred to as papers I and papers II respectively, (with their spin parameters set to zero) and they are all special cases of the general mathematical solution given by Ernst [14]. It is of primary importance that the parameters in the solution be related to a physical set of parameters in order for any subsequent analysis of the solution to have any significant physical meaning. For a physical set of parameters, one would prefer to use the individual masses and charges of each source and the distance between the sources. The invariant charge enclosed by a spacelike hypersurface can be found by the direct integration of Maxwell's equations. For space-times with a timelike Killing vector, a conserved quantity which can be interpreted as the contribution to the total mass from each body can be invariantly defined in analogy with the charge (See, for example, Refs. [9:15] and [16]). This paper follows Kramer [9] for 
the definition of the individual mass of each body. In Section $\mathbb{I}$, the integrals of charge and mass are given and they are applied to the Weyl-class solution for two Reissner-Nordström bodies in Section [II]. Section IV presents the solution for a parameterization of the nonWeyl-class double Reissner-Nordström solution based on the Weyl-class parameterization. These are then compared to the parameterizations proposed in papers I and II. It is shown that the parameterizations employed in papers I and II do not represent the physical masses or charges of the individual sources even in the Weyl-class limit (except for the special case of identical bodies in paper I). Due to the complexities of the parameterization, a rendering of the solution in terms of the individual masses and charges as given in Section $\square$ has not yet been accomplished. However, numerical analysis of the physical masses and charges is possible for a given set of parameters. In Section $\mathrm{V}$, balance without a strut or tension for numerical values of the physical mass and charge is examined. It is found that there are balance conditions for which neither body is critically charged and the Newtonian balance condition does not hold. This is in accordance with Bonnor's [1] test particle analysis. The dependence of the balance condition on the separation of the bodies is not yet known. A discussion of the results and conclusions are given in Sections $\nabla T$ and VII.

\section{MASS AND CHARGE}

For a static axially symmetric space-time, the mass $M_{i}$ and charge $Q_{i}$ of a source inside a closed 2-surface $\sigma_{i}$ are given by the integrals [17,9]

$$
\begin{aligned}
M_{i} & \equiv-\frac{1}{8 \pi} \oint_{\sigma_{i}} K^{a b} \sqrt{-g} d f_{a b}^{*} \\
Q_{i} & =-\frac{1}{8 \pi} \oint_{\sigma_{i}} F^{a b} \sqrt{-g} d f_{a b}^{*}
\end{aligned}
$$

where

$$
K^{a b} \equiv \xi^{a ; b}+\Phi F^{a b} .
$$

The timelike Killing vector is $\xi^{a}, F_{a b}$ is the electromagnetic field tensor, $\Phi$ is the electrostatic potential, $g$ is the determinant of the metric and $d f_{a b}^{*}$ is the dual to the surface element 2 -form $d f^{a b}$,

$$
d f_{a b}^{*}=\frac{1}{2} e_{a b c d} d f^{c d}
$$

(here $e_{a b c d}$ is the flat space Levi-Civita permutation symbol). The above integral conservation laws follow from the local conservation laws

$$
F_{; b ; a}^{a b}=0 \quad K_{; b ; a}^{a b}=0,
$$

the first, following from the conservation of charge and the second from the existence of the timelike Killing vector $\xi^{a}$ and the restriction to a static axially symmetric space-time metric. Since the Einstein-Maxwell equations also imply

$$
F_{; b}^{a b}=0 \quad K_{; b}^{a b}=0,
$$

in a source free region, any deformation of the surface $\sigma_{i}$ in the electrovacuum region outside the sources does not change the values of the integrals $M_{i}$ and $Q_{i}$. 


\section{THE WEYL-CLASS TWO BODY SOLUTION}

To investigate the structure of space-times with two sources, the Weyl-class double Reissner-Nordström solution provides a suitable yet mathematically uncumbersome framework from which to proceed. The solution is easily found through the method presented in Ref. 四. The metric for a static axially symmetric space-time can be written in the canonical form

$$
d s^{2}=e^{w} d t^{2}-e^{v-w}\left(d \rho^{2}+d z^{2}\right)-\rho^{2} e^{-w} d \phi^{2},
$$

where $w$ and $v$ are functions of the cylindrical coordinates $\rho$ and $z$. The Weyl-class solutions are characterized by the metric function $w$ being a function of the electrostatic potential, i.e. $w=w(\Phi)$ so that the gravitational and electrostatic equipotential surfaces overlap. For asymptotically flat boundary conditions, the unique functional relationship between $e^{w}$ and $\Phi$ is 18

$$
e^{w}=1-2 \frac{m_{\mathrm{T}}}{q_{\mathrm{T}}} \Phi+\Phi^{2},
$$

where $\Phi$ is the electrostatic potential and $m_{\mathrm{T}}$ and $q_{\mathrm{T}}$ are the total mass and charge respectively. The solution representing two "undercharged" $\left(M_{i}>\left|Q_{i}\right|\right)$ Reissner-Nordström bodies (or "black holes") is given by

$$
\Phi=a \frac{f-1}{a^{2} f-1},
$$

where

$$
\begin{aligned}
f=\left(\frac{R_{1}+R_{2}-2 l_{1}}{R_{1}+R_{2}+2 l_{1}}\right)\left(\frac{R_{3}+R_{4}-2 l_{2}}{R_{3}+R_{4}+2 l_{2}}\right), \\
R_{1}^{2} \equiv\left(z-d-2 l_{1}\right)^{2}+\rho^{2}, \\
R_{2}^{2} \equiv(z-d)^{2}+\rho^{2}, \\
R_{3}^{2} \equiv(z+d)^{2}+\rho^{2} \\
R_{4}^{2} \equiv\left(z+d+2 l_{2}\right)^{2}+\rho^{2} .
\end{aligned}
$$

The constant parameters $2 d$ and $2 l_{1}, 2 l_{2}$ are the coordinate distance between the horizons and the "lengths" of the horizons (Weyl "rods") respectively (see Fig. 1). The parameter $a$ is defined through the equation

$$
\frac{1+a^{2}}{a}=\frac{2 m_{\mathrm{T}}}{q_{\mathrm{T}}} .
$$

The metric function $e^{w}$ is found through Eq. (3.2). The metric function $e^{v}$ is

$$
e^{v}=\frac{\left(R_{1}+R_{2}\right)^{2}-4 l_{1}^{2}}{4 R_{1} R_{2}} \cdot \frac{\left(R_{3}+R_{4}\right)^{2}-4 l_{2}^{2}}{4 R_{3} R_{4}} \cdot\left[\frac{\left(\left(l_{1}+l_{2}+d\right) R_{1}+\left(l_{2}+d\right) R_{2}-l_{1} R_{4}\right) d}{\left(\left(l_{1}+d\right) R_{1}+R_{2} d-l_{1} R_{3}\right)\left(l_{2}+d\right)}\right]^{2} .
$$


Choosing the surface $\sigma_{1}$ to encompass body 1 and the surface $\sigma_{2}$ to encompass body 2 of Fig. 1, the mass and charge integrals of Eqs. (2.1) and (2.2) yield

$$
\begin{array}{ll}
M_{1}=\frac{1+a^{2}}{1-a^{2}} l_{1} & Q_{1}=\frac{2 a}{1-a^{2}} l_{1} \\
M_{2}=\frac{1+a^{2}}{1-a^{2}} l_{2} & Q_{2}=\frac{2 a}{1-a^{2}} l_{2}
\end{array}
$$

The above form of the individual mass and charge for each Reissner-Nordström body is similar to the form proposed in Ref. [4] for the mass and charge decomposition of two charged Curzon particles. It was stated in Ref. [7] that the conjectured charge decomposition for both the double Reissner-Nordström and Curzon cases were verified by direct calculation through Eq. (2.2). It is straightforward to verify that Eq. (2.1) yields the conjectured mass decomposition for the double Curzon solution. Because of the functional relationship between the gravitational potential and the electrostatic potential, not all of the parameters $M_{1}, M_{2}, Q_{1}, Q_{2}$ are independent. Thus the Weyl-class is also characterized by the constraint

$$
M_{1} Q_{2}=M_{2} Q_{1}
$$

Removal of the line singularity between the bodies yields Eq. (1.1) as an additional condition on the parameters. As a result, the parameters also satisfy Eq. (1.3). Thus equilibrium without a strut or tension occurs for "critically" charged sources and this balance is found to be independent of the separation distance [⿴囗十).

\section{NON-WEYL PARAMETERIZATIONS}

Generalizing the Weyl-class double Reissner-Nordström solution to the case in which the gravitational and electrostatic equipotential surfaces no longer overlap has usually been attempted through the means of generating techniques (see, for example, Refs. [6,7] and [9]). In these techniques, new solutions are generated from old ones rather than by solving the equations directly. Recently, considerable interest has focused upon a method [10] which constructs the Ernst potentials [11] from initial data on the symmetry axis. The complex Ernst potentials $\mathcal{E}(\rho, z)$ and $\Psi(\rho, z)$ of all stationary axisymmetric electrovacuum space-times with axis data of the form

$$
\mathcal{E}(z, \rho=0)=\frac{U-W}{U+W}, \quad \Psi(z, \rho=0)=\frac{V}{U+W},
$$

where

$$
\begin{gathered}
U=z^{2}+U_{1} z+U_{2} \\
V=V_{1} z+V_{2} \\
W=W_{1} z+W_{2}
\end{gathered}
$$


and $U_{1}, U_{2}, V_{1}, V_{2}, W_{1}, W_{2}$ are complex constants, have been found [14. However, a mathematical solution to the Einstein-Maxwell field equations does not imply a well understood physical interpretation of the solution. Sibgatullin's method of constructing the Ernst potentials aids in obtaining the physically meaningful parameterization which is sought for the two-body case in question.

In Sibgatullin's method, it is required that the Ernst potentials along the $z$-axis be specified. Our choice was [19,20]

$$
\begin{aligned}
& \mathcal{E}(\rho=0, z) \equiv e(z)=1-\frac{2\left(m_{1}\left(z+z_{2}\right)+m_{2}\left(z+z_{1}\right)\right)}{\left(z+z_{1}+m_{1}\right)\left(z+z_{2}+m_{2}\right)-q_{1} q_{2}}, \\
& \Psi(\rho=0, z) \equiv F(z)=\frac{q_{1}\left(z+z_{2}\right)+q_{2}\left(z+z_{1}\right)}{\left(z+z_{1}+m_{1}\right)\left(z+z_{2}+m_{2}\right)-q_{1} q_{2}} .
\end{aligned}
$$

It has the form of the Weyl-class double Reissner-Nordström axis data. If the additional Weyl-class constraint

$$
m_{1} q_{2}-m_{2} q_{1}=0
$$

is placed on the functions $e(z)$ and $F(z)$, then Sibgatullin's method yields the Weyl-class double Reissner-Nordström solution (in an alternate form to Ref. [4) and the parameters $m_{1}, m_{2}, q_{1}, q_{2}$ are the physical masses and charges as defined by Eqs. (2.1 2.2) (i.e., $M_{1}=$ $\left.m_{1}, Q_{1}=q_{1}, M_{2}=m_{2}, Q_{2}=q_{2}\right)$. For the solution of two Weyl-class Reissner-Nordström black holes (given in Section III), Fig. 1 shows the coordinate positions of the centers of the "rods" as $d+l_{1}$ for body 1 and $-d-l_{2}$ for body 2 . The parameters $z_{1}, z_{2}$ identify the negative of the coordinate positions of the centers of the "rods", i.e.,

$$
\begin{aligned}
& z_{1}=-d-l_{1}, \\
& z_{2}=d+l_{2} .
\end{aligned}
$$

If condition (4.6) is not imposed, $w \neq w(\Phi)$, i.e., the gravitational and electrostatic equipotential surfaces no longer overlap. In Section $\square$ it will be shown that the parameters $m_{1}, m_{2}, q_{1}, q_{2}$ then no longer carry the suggested physical meaning and the parameters $z_{1}, z_{2}$ no longer coincide with the centers of the "rods" when the Weyl-class constraint (4.6) is not imposed.

The full Ernst potentials $\mathcal{E}(\rho, z)$ and $\Psi(\rho, z)$ for the axis data of Eq. (4.5), expressed in terms of the cylindrical coordinates $(\rho, z)$, are found to be (the details of the method can be found in Refs. [10,12,21] and in the review article [22])

$$
\mathcal{E}=\frac{A-B}{A+B}, \quad \Psi=\frac{C}{A+B},
$$

where

$$
A \equiv \sum_{i<j}^{4} a_{i j} r_{i} r_{j}, \quad B \equiv \sum_{i=1}^{4} b_{i} r_{i}, \quad C \equiv \sum_{i=1}^{4} c_{i} r_{i}, \quad r_{n} \equiv \sqrt{\rho^{2}+\left(z-\alpha_{n}\right)^{2}}, \quad(n=1 \rightarrow 4) .
$$

The constants $\alpha_{n}$ in Eq. (4.7) are the roots of the equation 


$$
e(z)+[F(z)]^{2}=0
$$

and can only be real or complex conjugate pairs. The remaining constants $a_{i j}, b_{i}$ and $c_{i}$ are defined as follows:

$$
\begin{aligned}
& a_{i j} \equiv(-1)^{i+j+1} s_{i} s_{j} t_{i} t_{j}\left(s_{i} t_{j}-s_{j} t_{i}\right)\left|\begin{array}{cc}
s_{k} v_{k} & s_{l} v_{l} \\
t_{k} u_{k} & t_{l} u_{l}
\end{array}\right| \\
& (i<j ; k<l ; k, l \neq i, j ; \quad i, k=1 \rightarrow 3 ; j, l=2 \rightarrow 4) ; \\
& b_{i} \equiv(-1)^{i} s_{i} t_{i}\left(s_{i}-t_{i}\right)\left|\begin{array}{ccc}
s_{k}^{2} t_{k}^{2} & s_{l}^{2} t_{l}^{2} & s_{m}^{2} t_{m}^{2} \\
s_{k} v_{k} & s_{l} v_{l} & s_{m} v_{m} \\
t_{k} u_{k} & t_{l} u_{l} & t_{m} u_{m}
\end{array}\right| \\
& (k<l<m ; \quad k, l, m \neq i ; \quad i=1 \rightarrow 4 ; k=1,2 ; l=2,3 ; m=3,4) ; \\
& c_{i} \equiv(-1)^{i+1} s_{i} t_{i}\left(s_{i}-t_{i}\right)\left(K_{3} G_{i}+K_{4} H_{i}\right), \\
& G_{i} \equiv\left|\begin{array}{lll}
s_{k} t_{k}^{2} & s_{l} t_{l}^{2} & s_{m} t_{m}^{2} \\
s_{k} v_{k} & s_{l} v_{l} & s_{m} v_{m} \\
t_{k} u_{k} & t_{l} u_{l} & t_{m} u_{m}
\end{array}\right|, \quad H_{i} \equiv\left|\begin{array}{ccc}
s_{k}^{2} t_{k} & s_{l}^{2} t_{l} & s_{m}^{2} t_{m} \\
s_{k} v_{k} & s_{l} v_{l} & s_{m} v_{m} \\
t_{k} u_{k} & t_{l} u_{l} & t_{m} u_{m}
\end{array}\right|
\end{aligned}
$$

$(k<l<m ; k, l, m \neq i ; \quad i=1 \rightarrow 4 ; k=1,2 ; l=2,3 ; m=3,4)$

$$
\begin{gathered}
s_{i} \equiv \beta_{1}-\alpha_{i}, \quad t_{i} \equiv \beta_{2}-\alpha_{i} \\
u_{i} \equiv K_{1} s_{i} t_{i}+K_{3}^{2} t_{i}+K_{3} K_{4} s_{i}, \quad v_{i} \equiv K_{2} s_{i} t_{i}+K_{4}^{2} s_{i}+K_{3} K_{4} t_{i} \\
K_{1} \equiv \frac{m_{1} z_{2}+m_{2} z_{1}+\left(m_{1}+m_{2}\right) \beta_{1}}{\beta_{1}-\beta_{2}}, \quad K_{2} \equiv \frac{m_{1} z_{2}+m_{2} z_{1}+\left(m_{1}+m_{2}\right) \beta_{2}}{\beta_{2}-\beta_{1}} \\
K_{3} \equiv \frac{q_{1} z_{2}+q_{2} z_{1}+\left(q_{1}+q_{2}\right) \beta_{1}}{\beta_{1}-\beta_{2}}, \quad K_{4} \equiv \frac{q_{1} z_{2}+q_{2} z_{1}+\left(q_{1}+q_{2}\right) \beta_{2}}{\beta_{2}-\beta_{1}} \\
\beta_{1} \equiv-\frac{1}{2}\left(z_{1}+m_{1}+z_{2}+m_{2}-\sqrt{\left(z_{1}-z_{2}+m_{1}-m_{2}\right)^{2}+4 q_{1} q_{2}}\right) \\
\beta_{2} \equiv-\frac{1}{2}\left(z_{1}+m_{1}+z_{2}+m_{2}+\sqrt{\left(z_{1}-z_{2}+m_{1}-m_{2}\right)^{2}+4 q_{1} q_{2}}\right)
\end{gathered}
$$

where all of the subsequent quantities introduced are constants ultimately defined in terms of $m_{i}, q_{i}, z_{i}, i=1,2$, which specify the character and locations of the sources in the Weyl-class limit only. 
The expressions for $\mathcal{E}$ and $\Psi$ are in Kinnersley's [23] form and this permits one to write the corresponding metric functions as

$$
e^{w}=\frac{A \bar{A}-B \bar{B}+C \bar{C}}{(A+B)(\bar{A}+\bar{B})}, \quad e^{v}=\frac{A \bar{A}-B \bar{B}+C \bar{C}}{K_{0} r_{1} r_{2} r_{3} r_{4}},
$$

where

$$
K_{0}=\left(\sum_{i<j}^{4} a_{i j}\right)\left(\sum_{i<j}^{4} \bar{a}_{i j}\right)
$$

and a bar denotes complex conjugation. For a static metric, the electrostatic potential $\Phi$ is equal to the Ernst potential $\Psi$ and this completes the solution.

With the knowledge of the full Ernst potentials and the metric functions, the next step would be to evaluate the true mass and charge integrals in terms of the parameters $m_{1}, m_{2}, q_{1}, q_{2}, z_{1}, z_{2}$. It is to be stressed that outside of the Weyl-class, these parameters no longer carry the suggested physical meaning. For the metric (3.1), the integrals (2.1) and (2.2) can be written as relations in flat 3 -space $(i=1,2)$ :

$$
\begin{gathered}
M_{i}=\frac{1}{8 \pi} \oint_{\sigma_{i}} e^{-w} \mathcal{E}_{, \alpha} n^{\alpha} d A \\
Q_{i}=-\frac{1}{4 \pi} \oint_{\sigma_{i}} e^{-w} \Phi_{, \alpha} n^{\alpha} d A,
\end{gathered}
$$

where $n^{\alpha}$ ( $\alpha$ runs from 1 to 3 ) is the unit vector orthogonal to the surface and $d A$ denotes the invariant (flat) surface element (see also Ref. [9] and references therein).

We can extend the Weyl-class definitions of the coordinate positions of the bodies to the non-Weyl-class solution. There are three distinct types of sources of interest. They are characterized by the transition between a source with an event horizon to one without an event horizon. As mentioned previously, the constants $\alpha_{n}, n=1 \rightarrow 4$ in Eq. (4.7) are either real or complex conjugate pairs. By definition, we choose $\alpha_{1} \geq \alpha_{2}>\alpha_{3} \geq$ $\alpha_{4}$. A Reissner-Nordström "black hole" is characterized by real pairs of $\alpha_{n}$. Fig. 1 1 shows that, in the Weyl canonical coordinate system, $\alpha_{n}$ indicates the end points of a "Weyl rod", which itself is the event horizon surface. A "superextreme" object [12] or "naked singularity" is characterized by a complex conjugate pair of $\alpha_{n}$. Body 2 of Fig. 2 illustrates the manifestation of a "superextreme" body in the space-time. An "extreme" object, for example, would be characterized by real $\alpha_{n}$ for which $\alpha_{1}=\alpha_{2}$. Therefore we have the following definitions for the coordinate positions of the sources:

i) For a Reissner-Nordström "black hole", we define $-Z_{i}$ to be the coordinate position of the center of the "Weyl rod". For example, the coordinate position of body 1 of Fig. (2) is

$$
-Z_{1}=\frac{1}{2}\left(\alpha_{1}+\alpha_{2}\right) .
$$


ii) For a "superextreme" object, we define $-Z_{i}$ to be the coordinate position of the real part of $\alpha_{n}$. For example, body 2 of Fig. (2) is a "superextreme" object. Therefore its coordinate position is

$$
-Z_{2}=\operatorname{Re}\left(\alpha_{3}\right)=\operatorname{Re}\left(\alpha_{4}\right) .
$$

(One could consider the imaginary part of $\alpha_{n}$ as the end points of a "complex Weyl rod" with the coordinate position of this "complex rod" being defined as its intersection with the real axis ( $z$-axis).)

iii) For an "extreme object", we define $-Z_{i}$ to be the coordinate position of the point locating the zero "length" Weyl "rod". For example, if body 1 was an "extreme" object, then $\alpha_{1}=\alpha_{2}$ and $-Z_{1}=\alpha_{1}$.

We also define

$$
\operatorname{Re}\left(\alpha_{2}\right)>\operatorname{Re}\left(\alpha_{3}\right)
$$

as the condition for having two separated bodies irrespective of the type of object.

With the above integrals and the coordinate positions as defined above evaluated in terms of $m_{1}, m_{2}, q_{1}, q_{2}, z_{1}, z_{2}$, it would then be possible, in principle, to invert these equations and hence write the solution $(4.7-4.9)$ in terms of the true physical parameters $M_{i}, Q_{i}$ and the coordinate positions $Z_{i}, i=1,2$. Ideally, the coordinate positions of the sources should be replaced with the proper separation of the sources. The complexity of the above Ernst potentials makes the analytic evaluation of the integrals (4.12-4.13) and the proper separation difficult. As a consequence this goal has not yet been achieved. However, it is possible to numerically integrate Eqs. (2.1 2.2) for a given set $\left\{m_{1}, m_{2}, q_{1}, q_{2}, z_{1}, z_{2}\right\}$. This will prove to be useful in studying balance conditions without a strut in Section $\nabla$.

Although the numerical evaluation of the physical mass and charge can be achieved from the parameterizations of paper I or paper II, it was hoped that the parameterization proposed in this paper, based on the Weyl-class solution, would facilitate the analytic evaluation of the integrals. It is not difficult to show that the parameterizations in papers I or II do not correctly identify the individual masses and charges of each source. We stated earlier that our parameterization $\left\{m_{1}, m_{2}, q_{1}, q_{2}, z_{1}, z_{2}\right\}$ only represents the physical masses and charges and coordinate positions of each source when the Weyl-class condition (Eq. (3.2) or (4.6)) is imposed (i.e., $\left\{M_{1}=m_{1}, M_{2}=m_{2}, Q_{1}=q_{1}, Q_{2}=q_{2}, Z_{1}=z_{1}, Z_{2}=z_{2}\right\}$ ). We can best demonstrate the problems with the parameterizations of papers I and II by comparing the representation of a properly parameterized Weyl-class solution with each of the other parameterizations. Let the set $\left\{m_{1}, m_{2}, q_{1}, q_{2}, z_{1}, z_{2}\right\}$ represent the physical Weylclass parameters under the condition $m_{1} q_{2}=m_{2} q_{1}$. Then the relationships between the three parameterizations is found by solving the set of equations (setting the spin parameters found in papers I and II to zero) 


$$
\begin{aligned}
& \frac{\text { Weyl-class }}{m_{1}+m_{2}}=\frac{\text { Paper I }}{\tilde{m}_{1}+\tilde{m}_{2}}=\frac{\text { Paper II }}{\hat{m}_{1}+\hat{m}_{2}}
\end{aligned}
$$

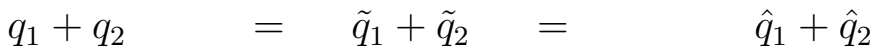

$$
\begin{aligned}
& z_{1}+z_{2}=\tilde{z}_{1}+\tilde{z}_{2}=\hat{z}_{1}+\hat{z}_{2} \\
& m_{1} z_{2}+m_{2} z_{1}=\tilde{m}_{1} \tilde{z}_{2}+\tilde{m}_{2} \tilde{z}_{1}=\hat{m}_{1} \hat{z}_{2}+\hat{m}_{2} \hat{z}_{1}+2 \hat{m}_{1} \hat{m}_{2} \\
& q_{1} z_{2}+q_{2} z_{1}=\tilde{q}_{1} \tilde{z}_{2}+\tilde{q}_{2} \tilde{z}_{1}=\hat{q}_{1} \hat{z}_{2}+\hat{q}_{2} \hat{z}_{1}+\hat{q}_{1} \hat{m}_{2}+\hat{q}_{2} \hat{m}_{1} \\
& z_{1} z_{2}+m_{1} m_{2}-q_{1} q_{2}=\tilde{z}_{1} \tilde{z}_{2}+\tilde{m}_{1} \tilde{m}_{2}=\quad \hat{z}_{1} \hat{z}_{2}-\hat{m}_{1} \hat{m}_{2} \text {. }
\end{aligned}
$$

The tilded and careted parameters are the parameterizations of papers I and II respectively. Table 1 summarizes the results of solving the system (4.15) given the values shown in column 1. The solution represents two Weyl-class Reissner-Nordström "critically charged" bodies without an intervening line singularity. It is clear that none of the parameter values in the latter two columns match the physical Weyl-class values. In fact one has to assign negative values to $\tilde{m}_{2}, \tilde{q}_{2}$ in order to obtain a positive physical mass and charge for source 2 . Thus, apart from one special case, neither the paper I nor the paper II parameterizations can be interpreted in as the invariant physical parameters. The only exception is for identical bodies (with or without a line singularity) in the parameterization of paper I. In this very special case of the Weyl-class, the parameters $\tilde{m}_{1}=\tilde{m}_{2}, \tilde{q}_{1}=\tilde{q}_{2}$ are the physical masses and charges. However, $\tilde{z}_{1}$ and $\tilde{z}_{2}$ do not identify the coordinate positions of the bodies as defined earlier. The paper II parameterization is not physical even for identical bodies.

It is the demand for the inclusion of the Weyl-class solution in Ref. [⿴囗 $\mid$ ] which led to our form of $e(z)$ and $F(z)$. It should be emphasized that our parameterization contains as a special case, the simplest clearly individually spherical two-body balance solution of two critically charged bodies. This can be best illustrated by examining the Simon 24,25 relativistic multipole moments of each parameterization. The first five Simon relativistic mass and charge multipole moments for our parameterization are

$$
\begin{aligned}
\mathcal{M}_{0}= & m_{1}+m_{2}, \\
\mathcal{M}_{1}= & -m_{1} z_{1}-m_{2} z_{2}, \\
\mathcal{M}_{2}= & m_{1} z_{1}^{2}+m_{2} z_{2}^{2}-\left(m_{1} m_{2}-q_{1} q_{2}\right)\left(m_{1}+m_{2}\right) \\
\mathcal{M}_{3}= & -m_{1} z_{1}^{3}-m_{2} z_{2}^{3}+\left(m_{1} m_{2}-q_{1} q_{2}\right)\left(2 m_{1} z_{1}+2 m_{2} z_{2}+z_{1} m_{2}+z_{2} m_{1}\right), \\
\mathcal{M}_{4}= & m_{1} z_{1}^{4}+m_{2} z_{2}^{4}-\left(m_{1} m_{2}-q_{1} q_{2}\right)\left[\left(m_{1}+m_{2}\right)\left(q_{1} q_{2}-m_{1} m_{2}\right)\right. \\
& +2\left(m_{1} z_{1}^{2}+m_{2} z_{2}^{2}\right)+\left(m_{1}+m_{2}\right)\left(z_{1}+z_{2}\right)^{2} \\
& \left.+\frac{1}{7}\left(m_{1}+m_{2}\right)\left(\left(q_{1}+q_{2}\right)^{2}-\left(m_{1}+m_{2}\right)^{2}\right)\right] \\
& -\frac{1}{210}\left(z_{1}-z_{2}\right)\left[16\left(z_{1}-z_{2}\right)\left(m_{1}+m_{2}\right)\left(m_{1} q_{2}-m_{2} q_{1}\right)^{2}\right. \\
& +z_{1}\left(30 m_{1}\left(m_{1} m_{2}+m_{2}^{2}-q_{2}^{2}\right)-3 q_{1}\left(3 m_{2} q_{1}+7 q_{2} m_{1}\right)\right) \\
& \left.-z_{2}\left(30 m_{2}\left(m_{1} m_{2}+m_{1}^{2}-q_{1}^{2}\right)-3 q_{2}\left(3 m_{1} q_{2}+7 q_{1} m_{2}\right)\right)\right]
\end{aligned}
$$

and 


$$
\begin{aligned}
\mathcal{Q}_{0}= & q_{1}+q_{2}, \\
\mathcal{Q}_{1}= & -q_{1} z_{1}-q_{2} z_{2}, \\
\mathcal{Q}_{2}= & q_{1} z_{1}^{2}+q_{2} z_{2}^{2}-\left(m_{1} m_{2}-q_{1} q_{2}\right)\left(q_{1}+q_{2}\right), \\
\mathcal{Q}_{3}= & -q_{1} z_{1}^{3}-q_{2} z_{2}^{3}+\left(m_{1} m_{2}-q_{1} q_{2}\right)\left(2 q_{1} z_{1}+2 q_{2} z_{2}+z_{1} q_{2}+z_{2} q_{1}\right) . \\
\mathcal{Q}_{4}= & q_{1} z_{1}^{4}+q_{2} z_{2}^{4}-\left(m_{1} m_{2}-q_{1} q_{2}\right)\left[\left(q_{1}+q_{2}\right)\left(q_{1} q_{2}-m_{1} m_{2}\right)\right. \\
& +2\left(q_{1} z_{1}^{2}+q_{2} z_{2}^{2}\right)+\left(q_{1}+q_{2}\right)\left(z_{1}+z_{2}\right)^{2} \\
& \left.+\frac{1}{7}\left(q_{1}+q_{2}\right)\left(\left(q_{1}+q_{2}\right)^{2}-\left(m_{1}+m_{2}\right)^{2}\right)\right] \\
& -\frac{1}{210}\left(z_{1}-z_{2}\right)\left[16\left(z_{1}-z_{2}\right)\left(q_{1}+q_{2}\right)\left(m_{1} q_{2}-m_{2} q_{1}\right)^{2}\right. \\
& -z_{1}\left(30 q_{2}\left(q_{1} q_{2}-m_{1} m_{2}+q_{1}^{2}\right)-3 m_{1}\left(13 m_{1} q_{2}-3 m_{2} q_{1}\right)\right) \\
& \left.+z_{2}\left(30 q_{1}\left(q_{1} q_{2}-m_{1} m_{2}+q_{1}^{2}\right)-3 m_{2}\left(13 m_{2} q_{1}-3 m_{1} q_{2}\right)\right)\right]
\end{aligned}
$$

respectively. In Newtonian physics, a system of two monopoles at positions $z_{1}, z_{2}$ has multipole moments

$$
\mathcal{M}_{n}=m_{1} z_{1}^{n}+m_{2} z_{2}^{n}, \quad \mathcal{Q}_{n}=q_{1} z_{1}^{n}+q_{2} z_{2}^{n} .
$$

It is interesting to observe that this is also the relativistic multipole structure for two Weylclass critically charged bodies, at least up to $\mathcal{M}_{4}, \mathcal{Q}_{4}$. There is an inherent asphericity imposed upon each, since the two bodies are interacting in a line. For non-linearly interacting sources in a line, one would not expect to realize perfect sphericity of the individual sources. (It is yet to be explained why the sphericity is maintained in the Weyl-class, at least up to $\mathcal{M}_{4}, \mathcal{Q}_{4}$.) Once the solution is written analytically in terms of the physically meaningful constants $M_{i}, Q_{i}$ and the coordinate positions $Z_{i}, i=1,2$, one will be able to examine the general multipole structure of non-linearly interacting spherical bodies.

For comparison, the first four Simon relativistic mass and charge multipole moments for the parameterization of paper I (with their spin parameters $a_{i}=0, i=1,2$ ) are

$$
\begin{aligned}
& \mathcal{M}_{0}=\tilde{m}_{1}+\tilde{m}_{2}, \\
& \mathcal{M}_{1}=-\tilde{m}_{1} \tilde{z}_{1}-\tilde{m}_{2} \tilde{z}_{2}, \\
& \mathcal{M}_{2}=\tilde{m}_{1} \tilde{z}_{1}^{2}+\tilde{m}_{2} \tilde{z}_{2}^{2}-\tilde{m}_{1} \tilde{m}_{2}\left(\tilde{m}_{1}+\tilde{m}_{2}\right) \\
& \mathcal{M}_{3}=-\tilde{m}_{1} \tilde{z}_{1}^{3}-\tilde{m}_{2} \tilde{z}_{2}^{3}+\tilde{m}_{1} \tilde{m}_{2}\left(2 \tilde{m}_{1} \tilde{z}_{1}+2 \tilde{m}_{2} \tilde{z}_{2}+\tilde{z}_{1} \tilde{m}_{2}+\tilde{z}_{2} \tilde{m}_{1}\right)
\end{aligned}
$$

and

$$
\begin{aligned}
& \mathcal{Q}_{0}=\tilde{q}_{1}+\tilde{q}_{2}, \\
& \mathcal{Q}_{1}=-\tilde{q}_{1} \tilde{z}_{1}-\tilde{q}_{2} \tilde{z}_{2}, \\
& \mathcal{Q}_{2}=\tilde{q}_{1} \tilde{z}_{1}^{2}+\tilde{q}_{2} \tilde{z}_{2}^{2}-\tilde{m}_{1} \tilde{m}_{2}\left(\tilde{q}_{1}+\tilde{q}_{2}\right) \\
& \mathcal{Q}_{3}=-\tilde{q}_{1} \tilde{z}_{1}^{3}-\tilde{q}_{2} \tilde{z}_{2}^{3}+\tilde{m}_{1} \tilde{m}_{2}\left(2 \tilde{q}_{1} \tilde{z}_{1}+2 \tilde{q}_{2} \tilde{z}_{2}+\tilde{z}_{1} \tilde{q}_{2}+\tilde{z}_{2} \tilde{q}_{1}\right) .
\end{aligned}
$$

The first four Simon relativistic mass and charge multipole moments for the parameterization of paper II (with their spin parameters $a_{i}=0, i=1,2$ ) are

$$
\begin{aligned}
\mathcal{M}_{0}= & \hat{m}_{1}+\hat{m}_{2}, \\
\mathcal{M}_{1}= & -\hat{m}_{1} \hat{z}_{1}-\hat{m}_{2} \hat{z}_{2}+2 \hat{m}_{1} \hat{m}_{2}, \\
\mathcal{M}_{2}= & \hat{m}_{1} \hat{z}_{1}^{2}+\hat{m}_{2} \hat{z}_{2}^{2}+\hat{m}_{1} \hat{m}_{2}\left(\hat{m}_{1}+\hat{m}_{2}-2 \hat{z}_{1}-2 \hat{z}_{2}\right), \\
\mathcal{M}_{3}= & -\hat{m}_{1} \hat{z}_{1}^{3}-\hat{m}_{2} \hat{z}_{2}^{3} \\
& +\hat{m}_{1} \hat{m}_{2}\left(2 \hat{m}_{1} \hat{m}_{2}+2 \hat{z}_{1} \hat{z}_{2}+2 \hat{z}_{1}^{2}+2 \hat{z}_{2}^{2}-\hat{m}_{1} \hat{z}_{2}-\hat{m}_{2} \hat{z}_{1}-2 \hat{m}_{1} \hat{z}_{1}-2 \hat{m}_{2} \hat{z}_{2}\right)
\end{aligned}
$$


and

$$
\begin{aligned}
\mathcal{Q}_{0}= & \hat{q}_{1}+\hat{q}_{2}, \\
\mathcal{Q}_{1}= & -\hat{q}_{1} \hat{z}_{1}-\hat{q}_{2} \hat{z}_{2}+\hat{m}_{1} \hat{q}_{2}+\hat{m}_{2} \hat{q}_{1}, \\
\mathcal{Q}_{2}= & \hat{q}_{1} \hat{z}_{1}^{2}+\hat{q}_{2} \hat{z}_{2}^{2}+\hat{m}_{1} \hat{m}_{2}\left(\hat{q}_{1}+\hat{q}_{2}\right)-\left(\hat{q}_{1} \hat{m}_{2}+\hat{q}_{2} \hat{m}_{1}\right)\left(\hat{z}_{1}+\hat{z}_{2}\right), \\
\mathcal{Q}_{3}= & -\hat{q}_{1} \hat{z}_{1}^{3}-\hat{q}_{2} \hat{z}_{2}^{3}-\hat{m}_{1} \hat{m}_{2}\left(2 \hat{q}_{1} \hat{z}_{1}+2 \hat{q}_{2} \hat{z}_{2}+\hat{z}_{1} \hat{q}_{2}+\hat{z}_{2} \hat{q}_{1}\right) \\
& +\left(\hat{q}_{1} \hat{m}_{2}+\hat{q}_{2} \hat{m}_{1}\right)\left(\hat{m}_{1} \hat{m}_{2}+\hat{z}_{1} \hat{z}_{2}+\hat{z}_{1}^{2}+\hat{z}_{2}^{2}\right) .
\end{aligned}
$$

If the above parameterizations did represent the physical mass and charge, it is evident that the multipole structure would not be that of Newtonian spherical bodies even for critically charged bodies. As stated earlier, it should be noted that in the parameterization of paper I, it can be shown that only in the case of identical bodies, the parameters $\tilde{m}_{1}=\tilde{m}_{2}, \tilde{q}_{1}=\tilde{q}_{2}$ are the physical mass and charge. However, in this case the multipoles still do not have the form of Eq. (4.18) since the parameters $\tilde{z}_{1}$ and $\tilde{z}_{2}$ do not identify the positions of the bodies as defined earlier. A simple transformation would correct the multipoles in this case.

\section{THE EQUILIBRIUM CONDITION}

In order for the space-time to be regular on the $z$-axis between the sources (removal of the Weyl line singularity or imposition of the condition for elementary flatness [26]), it is required that the metric function

$$
v(z, \rho=0)=0
$$

between the sources. If the origin of the coordinate system is located between the sources (i.e., $\operatorname{Re}\left(\alpha_{2}\right)>0, \operatorname{Re}\left(\alpha_{3}\right)<0$ ), then application of Eq. (5.1), after some simplification, yields the balance equation

$$
K \equiv \frac{a_{12}\left(\bar{a}_{13}+\bar{a}_{14}\right)+\bar{a}_{12}\left(a_{13}+a_{14}\right)}{K_{0}}=0 .
$$

Three cases were examined: i) Two Reissner-Nordström black holes, ii) Two ReissnerNordström superextreme bodies and iii) One black hole and one superextreme body.

The procedure for testing for equilibrium without an intervening strut or tension will be as follows:

1. Assign numerical values to five of the six parameters from the unphysical set $\left\{m_{1}, m_{2}, q_{1}, q_{2}, z_{1}, z_{2}\right\}$.

2. Solve Eq. (5.2) for the unknown variable.

3. If a real root of Eq. (5.2) exists, then evaluate Eqs. (2.1) and (2.2) to determine the physical mass and charge parameters.

The results for each of the three cases are as follows: 


\section{A. Two Reissner-Nordström Black Holes}

Numerous sets of the parameters $\left\{m_{1}, m_{2}, q_{1}, q_{2}, z_{1}, z_{2}\right\}$, such that the constants $\alpha_{n}, n=$ $1 \rightarrow 4$ are real, were investigated. No roots were found of Eq. (5.2). For example, choosing $m_{1}=9.0, \quad q_{1}=3.0, \quad z_{1}=-15.0, \quad m_{2}=8.0, \quad q_{2}=2.0$, no balance for $0 \leq z_{2} \leq 10^{10}$ was found. These findings are consistent with other results [6,8, 15] that two Reissner-Nordström black holes cannot be found in equilibrium without an intervening strut or tension.

\section{B. Two Reissner-Nordström Superextreme Bodies}

Numerous sets of the parameters $\left\{m_{1}, m_{2}, q_{1}, q_{2}, z_{1}, z_{2}\right\}$, such that the constants $\alpha_{n}, n=$ $1 \rightarrow 4$ are complex conjugate pairs, were investigated. No roots were found of Eq. (5.2). For example, in choosing $m_{1}=3.0, q_{1}=9.0, \quad z_{1}=-15.0, m_{2}=2.0, q_{2}=8.0$, no balance for $0 \leq z_{2} \leq 10^{10}$ was found. These findings suggest that two Reissner-Nordström superextreme bodies cannot be found in equilibrium without a strut or tension.

\section{One Black Hole and One Superextreme Body}

The following three different cases were found for which Eq. (5.2) has a real root. Each case has the configuration illustrated in Fig. 2.

Case A) For $m_{1}=6.0, \quad q_{1}=2.0, \quad z_{1}=-5.0, \quad m_{2}=-0.7, \quad q_{2}=4.0, \quad$ balance at approximately [27] $z_{2}=2.08$ was found. The values of $\alpha_{n}$ are $\alpha_{1}=10.3, \quad \alpha_{2}=$ 1.74, $\alpha_{3}=-3.11+i 4.30, \quad \alpha_{4}=-3.11-i 4.30$. Using Eqs. (4.12 4.13), the physical masses and charges are $M_{1}=3.95, \quad Q_{1}=-0.887, \quad M_{2}=1.35, \quad Q_{2}=6.89$. Using the definitions of coordinate positions described in Section IV, it was found that $Z_{1}=-6.03$ and $Z_{2}=3.11$. Thus balance has occurred for $M_{1} M_{2}>Q_{1} Q_{2}$, $Q_{1} Q_{2}<0$ at a coordinate separation of $\mathcal{S} \equiv Z_{2}-Z_{1}=9.13$. Note that the parameter $m_{2}$ is negative but both physical masses are positive. The parameterizations of papers I and II yield respectively

$$
\begin{array}{rlrl}
\text { Paper I } & & \text { Paper II } \\
\tilde{m}_{1}=4.96 & \hat{m}_{1}=4.36 \\
\tilde{q}_{1}=2.31 & \hat{q}_{1}=-1.05 \\
\tilde{m}_{2}=0.34 & \hat{m}_{2}=0.94 \\
\tilde{q}_{2}=3.69 & \hat{q}_{2}=7.05 \\
\tilde{z}_{1}=-6.60 & \hat{z}_{1}=-6.00 \\
\tilde{z}_{2}=3.68 & \hat{z}_{2}=3.08
\end{array}
$$

which do not agree with the integrated values of Eqs. (2.1) and (2.2). This demonstrates that in general none of the analytic parameterizations proposed, including our own, are suitable choices for the individual masses and charges of the sources.

Case B) For $m_{1}=9.0, \quad q_{1}=3.0, \quad z_{1}=-40.0, \quad m_{2}=2.5, \quad q_{2}=8.0, \quad$ balance was found at approximately $z_{2}=34.6$. The values of $\alpha_{n}$ are $\alpha_{1}=48.4, \quad \alpha_{2}=$ 
$31.61, \quad \alpha_{3}=-34.62+i 7.65, \quad \alpha_{4}=-34.62-i 7.65$. The physical masses and charges are $M_{1}=8.87, \quad Q_{1}=2.00, \quad M_{2}=2.63, \quad Q_{2}=9.00$. The coordinate positions are $-Z_{1}=40.01,-Z_{2}=-34.6$ Thus balance has occurred for $M_{1} M_{2}>Q_{1} Q_{2}, Q_{1} Q_{2}>0$ at a coordinate separation of $\mathcal{S}=74.6$.

Case C) For $m_{1}=900.0, \quad q_{1}=300.0, \quad z_{1}=-865.0, \quad m_{2}=0.025, \quad q_{2}=0.080, \quad$ balance was found at approximately $z_{2}=21.581$. The values of $\alpha_{n}$ are $\alpha_{1}=1713.5, \alpha_{2}=$ $16.474, \quad \alpha_{3}=-21.582+i 0.26226, \quad \alpha_{4}=-21.582-i 0.26226$. The physical masses and charges are $M_{1}=899.71, \quad Q_{1}=298.25, \quad M_{2}=0.31897, \quad Q_{2}=1.8254$. The coordinate positions are $-Z_{1}=865.00,-Z_{2}=-21.582$ Thus balance has occurred for $Q_{1} Q_{2}>M_{1} M_{2}, Q_{1} Q_{2}>0$ at a coordinate separation of $\mathcal{S}=886.58$.

\section{Comparison with Test Particle Analysis}

Bonnor's [1] examination of a test particle in the field of a Reissner-Nordström source yielded a wide variety of balance conditions. The following cases for separation-independent equilibrium were examined (note: $M, Q$ characterize the Reissner-Nordström space-time and $m, q$ are the test body parameters):

Case 1) For $q=\epsilon m, Q=\eta M, \epsilon, \eta= \pm 1$, balance occurs if $\epsilon=\eta$.

Case 2) If $m=|q|, \quad M \neq|Q|$, or $m \neq|q|, \quad M=|Q|$, no equilibrium is possible.

Case 3) If $m M=q Q$ but $m \neq|q|$, then no equilibrium is possible.

Since the exact solution under study contains the Weyl-class solution as a special case, we also find Bonnor's case 1 as a separation-independent equilibrium condition. Case 2 or 3 cannot be tested readily by our numerical procedure. In order to do so, one would have to have the good fortune of correctly choosing the set $\left\{m_{1}, m_{2}, q_{1}, q_{2}, z_{1}, z_{2}\right\}$ such that the physical masses and charges satisfy the given conditions (i.e. $M_{1}=\left|Q_{1}\right|$ etc.). Then, to test the dependence on separation, one would need to choose a new set of unphysical parameters such that the proper separation changes while the physical masses and charges remain the same.

The following separation-dependent cases were also found in Ref. [1]:

Case 4) If $|Q|>M, \quad m M=-q Q$ and $m^{2} \neq q^{2}$ with $q Q<0$, then an equilibrium exists at

$$
r=\frac{Q^{2}}{2 M}
$$

Case 5) If $|Q|>M,|q|<m, q Q<0$ or

Case 6) if $|Q|>M,|q|<m, q Q>0, q Q<m M$ then an equilibrium position exists at

$$
r=\frac{Q^{2}\left(M\left(m^{2}-q^{2}\right)+q \sqrt{\left(m^{2}-q^{2}\right)\left(Q^{2}-M^{2}\right)}\right)}{m^{2} M^{2}-q^{2} Q^{2}} .
$$


Case 7) If $|Q|<M,|q|>m, q Q>0, q Q>m M$ then an equilibrium position exists at

$$
r=\frac{Q^{2}\left(M\left(m^{2}-q^{2}\right)-q \sqrt{\left(m^{2}-q^{2}\right)\left(Q^{2}-M^{2}\right)}\right)}{m^{2} M^{2}-q^{2} Q^{2}} .
$$

Thus we have found a direct correspondence between cases A-C of the exact solution and cases 5-7 of Bonnor's test particle analysis. The separation dependence of cases 4-7 cannot be studied in the exact solution using the present methods for the same reasons cases 2-3 cannot be studied. Since the separation dependence cannot be tested using the present methods, there is little value in numerically calculating the proper separation of the sources in cases $\mathrm{A}-\mathrm{C}$.

The physical parameters in case $\mathrm{C}$ could approximate a test body in a strong gravitational field. Using these values in case 7 and transforming from spherical coordinates to cylindrical coordinates for a single Reissner-Nordström body using the transformation (with $\theta=0$ )

$$
\begin{aligned}
& z=(r-M) \cos \theta, \\
& \rho=\sqrt{r^{2}-2 M r+Q^{2}} \sin \theta,
\end{aligned}
$$

Bonnor's method yields a coordinate separation of $\mathcal{S}=1465.5$. Since the separation of the bodies from these two methods are not consistent, it would appear that case $\mathrm{C}$ does not sufficiently approximate a test body.

\section{DISCUSSION}

The essential departure in the present paper from previous work is the attempt to parameterize the solution in terms of true physical constants of the space-time. For a static axially symmetric solution of the Einstein-Maxwell equations, the integrals of Eq. (2.1) and (2.2) provide the invariant parameters required for meaningful analysis of the properties of the solution.

There are three cases of the exact solution which have not been examined. They are an extreme body with respectively a Reissner-Nordström black hole, a superextreme body, and another extreme body for which the solution is not of the Weyl-class. Knowledge of the solution analytically in terms of the physical parameters is required to analyze these cases adequately.

Ref. [1] defines the terms "undercharged", "overcharged" and "critically charged" as follows $(i=1,2)$ :

$$
\begin{array}{ll}
M_{i}^{2}>Q_{i}^{2} & \text { "undercharged" } \\
M_{i}^{2}<Q_{i}^{2} & \text { "overcharged" } \\
M_{i}^{2}=Q_{i}^{2} & \text { "critically charged" }
\end{array}
$$

For the Weyl-class, the "lengths" of the Weyl rods are [4] $2 l_{i}=2 \sqrt{M_{i}^{2}-Q_{i}^{2}}, i=1,2$. If body 1 is "critically charged" [28], then $\alpha_{1}=\alpha_{2}(=d)$ since $l_{1}=0$ (see Fig. (1). This implies that the terminology "critically charged" body and "extreme" body may be used interchangeably 
for Weyl-class solutions. If body 1 is "undercharged", $\alpha_{1}\left(=d+2 l_{1}\right)$ and $\alpha_{2}(=d)$ are real quantities. Thus "undercharged body" and "black hole" are synonymous terms in the Weylclass. Finally, if body 1 is "overcharged", $\alpha_{1}\left(=d+l_{1}\right)$ and $\alpha_{2}\left(=d+\bar{l}_{1}\right)$ are complex conjugates. Thus the terms "overcharged" and "superextreme" are equivalent descriptions in the Weyl-class. Unlike the Weyl-class solutions where the "lengths" of the Weyl rods (real or complex) depend only upon the mass and charge of that source, it is strongly suggested from the analysis of Section $\nabla$ that for the general (non-Weyl-class) solution, the "lengths" of the rods also depend on the mass and charge of the other source and the distance separating the bodies as well. It would thus be possible to have a "critically" charged body (according to Eq. (6.3)) for which the "rod" is either of non-zero "length" or "complex". This is important in terms of nomenclature for describing the physics of the space-time. Since the transition of a pair (eg. $\left.\left(\alpha_{1}, \alpha_{2}\right)\right)$ from real values to a complex conjugate pair in Sibgatullin's [10 method defines a differentiation of an object with a horizon to one without, it would seem that the appropriate description would be respectively, a black hole (horizon), "extreme" body (zero "length" Weyl rod) and "superextreme" body (no horizon or naked singularity) as described in paper I. The descriptions "under", "over" and "critically" charged body should be reserved for the relations $M_{i}^{2}>Q_{i}^{2}, M_{i}^{2}<Q_{i}^{2}$ and $M_{i}^{2}=Q_{i}^{2}$ respectively between the individual masses and charges. This classification scheme would describe equilibrium conditions more precisely once all are identified. The appropriateness of such a scheme would become apparent when the analytic physical parameterization of the solution is known.

Bonnor's [1] test particle analysis has been modified [30] in such a way that the equilibrium conditions of a charged test particle in the field of a Kerr-Newman source can be studied. The generalization of the mathematical solution to two spinning sources (Kerr-Newman sources) is already known 12, 13]. One is able to invariantly define angular momentum for a stationary space-time in a manner similar to Eqs. (2.1 2.2) because of the presence of a spacelike Killing vector (rotational symmetry) (see Ref. [15] and references therein for definitions of mass and angular momentum of stationary vacuum fields). It is unknown how the subsequent analysis of two identical spinning bodies in paper I based on the invariant definitions will affect their results, if at all. However, it is clear that the parameterization given is inadequate for the physical analysis of the general case (non-identical bodies).

\section{CONCLUSIONS}

The solution derived in papers I, II and this paper is a generalization of the Weyl-class double Reissner-Nordström solution. However, the analytic parameterizations presented in papers I, II and this paper cannot in all cases be interpreted as the true physical constants of the spacetime. The invariant physical charge for each source is found by direct integration of Maxwell's equations. The physical mass is invariantly defined [9] in a manner similar to which the charge was found. Numerical methods were used to evaluate the invariant individual masses and charges for the axially symmetric superposition of two Reissner-Nordström bodies. It was found that neither the Newtonian balance condition nor critically charged bodies are necessary for electrostatic equilibrium. The dependence of the balance condition on the separation of the bodies is not yet known due to the complexities involved in expressing the solution analytically in terms of the true physical set of parameters. However, all the balance conditions found are consistent with Bonnor's test particle analysis. This suggests 
that there exist equilibrium conditions which depend on the separation of the sources. The parameterization of this paper is manifestly physical in the Weyl-class limit.

\section{ACKNOWLEDGMENTS}

This research was supported, in part, by a grant from the Natural Sciences and Engineering Research Council of Canada and a Natural Sciences and Engineering Research Council Postgraduate Scholarship (GPP). 


\section{REFERENCES}

[1] W.B. Bonnor, Class. Quantum Grav. 10, 2077 (1993).

[2] B.M. Barker and R.F. O'Connell, Phys. Lett. A 61, 297 (1977).

[3] T. Kimura and T. Ohta, Phys. Lett. 63A, 193 (1977).

[4] F.I. Cooperstock and V. de la Cruz, Gen. Rel. Grav. 10, 681 (1979).

[5] A. Papapetrou, Proc. R. Irish Acad. 51, 191 (1947); S.M. Majumdar, Phys. Rev. 72, 390 (1947).

[6] A. Tomimatsu, Prog. Theor. Phys. 71, 409 (1984).

[7] J. Carminati and F.I Cooperstock, Class. Quantum Grav. 8, L171 (1991); Gen. Rel. Grav. 24, 881 (1992).

[8] G.P. Perry, in Proceedings of the 5th Canadian Conference on General Relativity and Relativistic Astrophysics, R.B. Mann and R.G. McLenaghan eds., (World Scientific, Singapore, 1994) p. 449.

[9] D. Kramer, Class. Quantum Grav. 5, 1435 (1988).

[10] N.R. Sibgatullin, Oscillations and Waves in Strong Gravitational and Electromagnetic Fields (Nauka, Moskow, 1984), in Russian [English translation: Springer-Verlag, Berlin, 1991].

[11] F.J. Ernst, Phys. Rev. 168, 1415 (1968).

[12] V.S. Manko, J. Martín, and E. Ruiz, J. Math. Phys. 35, 6644 (1994).

[13] A. Chamorro, V.S. Manko and N.R. Sibgatullin, Lect. Notes Phys. 423, 119 (1993).

[14] F.J. Ernst, Phys. Rev. D 50, 4993; 6179 (1994).

[15] W. Dietz and C. Hoenselaers, Ann. Phys. (USA) 165, 319 (1985).

[16] H. Stephani, General Relativity, (Cambridge University Press, Cambridge, 1990), 2nd ed., p. 208

[17] Notations and conventions used are those of L. D. Landau and E. M. Lifshitz, The Classical Theory of Fields (Pergamon Press, Oxford, 1975), 4th revised English ed.

[18] H. Weyl, Ann. Phys. (Leipzig), 54, 117 (1917).

[19] G.P. Perry and F.I. Cooperstock, in Proceedings of the 14th International Conference on General Relativity and Gravitation, Florence, Italy, Aug 1995.

[20] G.P. Perry and F.I. Cooperstock, in Proceedings of the 6th Canadian Conference on General Relativity and Relativistic Astrophysics, Fredericton, Canada, May 1996.

[21] V.S. Manko and N.R. Sibgatullin, Phys. Lett. A 168, 343 (1992); J. Math. Phys. 34, 170 (1993).

[22] V.S. Manko and N.R. Sibgatullin, Class. Quantum Grav. 10, 1390 (1993).

[23] W. Kinnersley, J. Math. Phys. 14, 651 (1973).

[24] W. Simon, J. Math. Phys. 25, 1035 (1984).

[25] C. Hoenselaers and Z. Perjés, Class. Quantum Grav. 7, 1819 (1990).

[26] J. L. Synge, Relativity: The General Theory, (North Holland, Amsterdam, 1966), pp. 313.

[27] In cases A-C, Eq. (5.2) has been solved to a precision of $|K|<10^{-50}$ using highly refined values of $z_{2}$.

[28] It should be noted that having identical roots $\alpha_{1}=\alpha_{2}$ is not sufficient for identifying critically charged bodies even in the Weyl-class. The Curzon particle is such an object with $\alpha_{1}=\alpha_{2}$ but it is not necessarily critically charged. See Refs. 229] and [4].

[29] H. E. J. Curzon, Proc. Lond. Math. Soc. 23, 477 (1924). 
[30] Aguirregabiria J M, Chamorro A, Suinaga J and Vishveshwara C V 1995 Class. Quantum Grav. 12699 


\section{FIGURES}

FIG. 1. Schematic of two Reissner-Nordström black holes in cylindrical coordinates. The thick lines are the Weyl "rods" which show the locations of the event horizon surfaces.

FIG. 2. Schematic of a Reissner-Nordström black hole and a Reissner-Nordström superextreme body. The dotted line is a "complex Weyl rod". The intersection of the "rod" with the $z$-axis is defined as the coordinate position of body 2 . 


\section{TABLES}

TABLE I. The values of the parameters in the parameterizations of papers I and II is shown given the Weyl-Class values.

\begin{tabular}{ccc}
\hline \hline Weyl-Class & Paper I & Paper II \\
\hline$m_{1}=8$ & $\tilde{m}_{1}=14.17$ & $\hat{m}_{1}=3.58$ \\
$q_{1}=8$ & $\tilde{q}_{1}=14.17$ & $\hat{q}_{1}=3.58$ \\
$m_{2}=3$ & $\tilde{m}_{2}=-3.17$ & $\hat{m}_{2}=7.42$ \\
$q_{2}=3$ & $\tilde{q}_{2}=-3.17$ & $\hat{q}_{2}=7.42$ \\
$z_{1}=-7$ & $\tilde{z}_{1}=-2.02$ & $\hat{z}_{1}=-4.7$ \\
$z_{2}=7$ & $\tilde{z}_{2}=2.02$ & $\hat{z}_{2}=4.7$ \\
\hline \hline
\end{tabular}




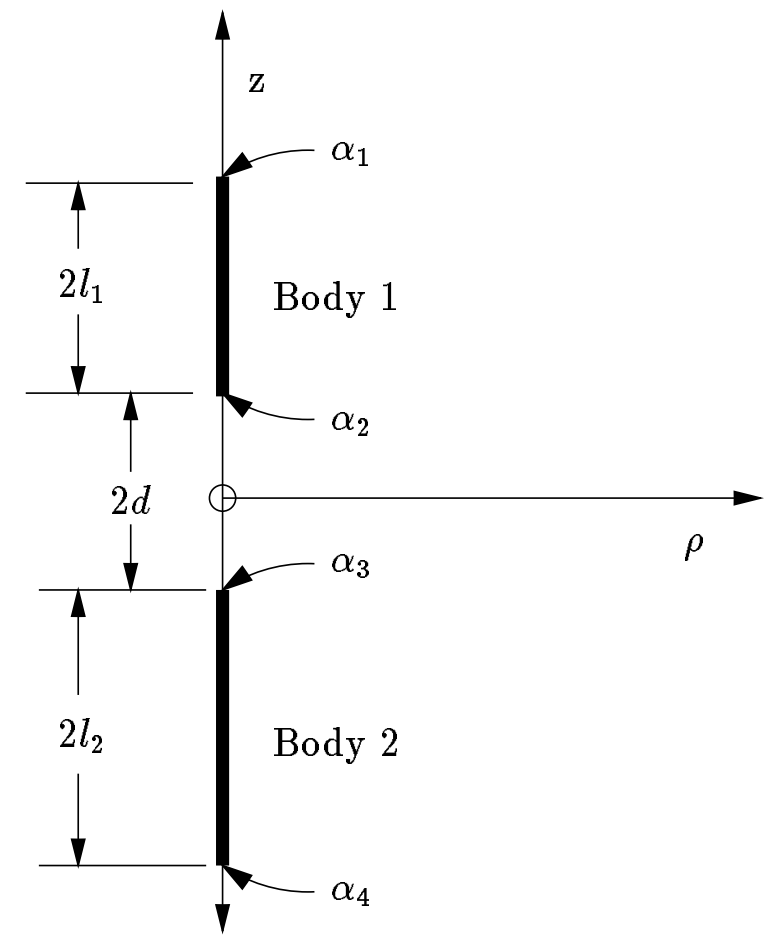

Figure 1: Schematic of two Reissner-Nordström black holes in cylindrical coordinates. The thick lines are the Weyl "rods" which show the locations of the event horizon surfaces. 


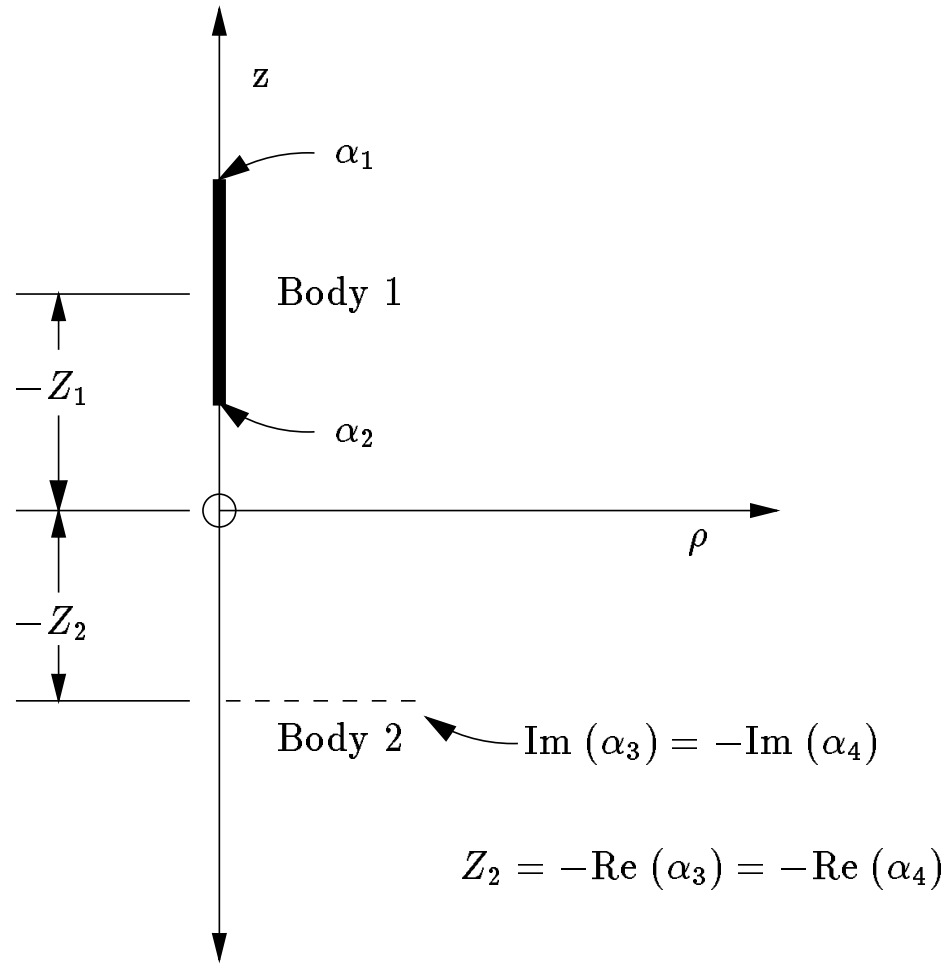

Figure 2: Schematic of a Reissner-Nordström black hole and a ReissnerNordström superextreme body. The dotted line is a "complex Weyl rod". The intersection of the "rod" with the $z$-axis is defined as the coordinate position of body 2 . 